\title{
PRIMARY REPAIR OF RUPTURE OF A MAIN AND LOBAR BRONCHUS
}

\author{
Filip E. Muysoms, MD, and Henry A. Van Swieten, MD, PhD, Nieuwegein, The Netherlands
}

A 16-year-old boy was transferred from another hospital to our department because of clinical suspicion of a bronchial rupture after blunt chest trauma. He had been in a traffic accident as the driver of a small motorcycle. On admission he was intubated, nonsedated, and anxious. He had hypotension, tachycardia, and massive subcutaneous emphysema over the chest, the upper part of the abdomen, and in the neck. A small thoracic catheter was in place. A roentgenogram of the chest made in the referring hospital showed a complete pneumothorax on the right side with extensive subcutaneous and mediastinal emphysema. He had a stable pelvic fracture, confirmed by radiography.

The patient was sedated. An arterial pressure line, a jugular catheter, and a larger thoracic drain were placed. A major air leak was detected. A flexible bronchoscope, inserted in the emergency department, revealed a large defect of the right main bronchus just distal to the carina. The patient was then taken to the operating room for urgent thoracotomy. After placement of a single-lumen endotracheal tube into the left main bronchus, the patient was positioned on his left side and a right posterolateral thoracotomy above the fifth rib was performed. The right lung had collapsed and the right upper lobe contained a large intrapulmonary hematoma. A complex, double bronchial rupture was present-a complete transection of the right main bronchus and an incomplete rupture of the right lower lobe bronchus. The cartilaginous portion of the main bronchus was transected transversely about $1 \mathrm{~cm}$ distal to the carina. The membranous portion had ruptured transversely more proximally at the level of the carina. The two transection planes were connected by a double longitudinal tear at the junction between the cartilaginous and membranous parts of the right main bronchus (Fig. 1). The right lower lobar bronchus had an incomplete transverse rupture about half the circumference of the bronchus and $0.5 \mathrm{~cm}$ distal to the ostium of the bronchus for the right middle lobe. Both ruptures were

From the Department of Cardiopulmonary Surgery, St. Antonius Ziekenhuis Koekoekslaan 1, 3435 CM Nieuwegein, The Netherlands.

Received for publication August 12, 1996; accepted for publication August 26, 1996.

Address for reprints: Filip Muysoms, MD, Emiel Clauslaan 133, 9800 Astene-Deinze, Belgium.

J Thorac Cardiovasc Surg 1997;113:415-7

Copyright (C 1997 by Mosby-Year Book, Inc.

$0022-5223 / 97 \$ 5.00+0 \quad \mathbf{1 2 / 5 4 / 7 7 5 7 2}$ primarily repaired. Interrupted resorbable Vicryl 3-0 sutures were used for the cartilaginous part. The membranous part of the main bronchial rupture was repaired with three continuous 5-0 polypropylene sutures. Care was taken to minimize peribronchial dissection so as to avoid compromising bronchial vascularization. After the repair, a minor air leak from the main bronchial suture was accepted. The sutures were covered with parietal pleura and fibrin glue. After the operation, the patient was supported with a ventilator for 6 days; the thoracic drain was removed after 5 days. Further recovery was uneventful, and after rehabilitation for the pelvic fracture the patient was discharged 30 days after admission. A roentgenogram of the chest showed good expansion of the right lung and a resorbing hematoma of the right upper lobe (Fig. 2). A follow-up bronchoscopic study after 5 months showed good patency of the main and lobar bronchi. On radioscopy the hematoma of the right upper lobe had disappeared.

Tracheobronchial ruptures are most frequently caused by forceful trauma like motor vehicle accidents. They are rather uncommon and probably have a high prehospital mortality. ${ }^{1,2}$ Symptoms of bronchial rupture include dyspnea, subcutaneous emphysema, and pneumothorax. Associated injuries are very frequent in these patients.

For diagnosis, bronchoscopy is the method of first choice. Bronchoscopy should be used liberally in injured patients in whom bronchial rupture is suspected. ${ }^{2}$

Roentgenographic signs of rupture of a major airway are subcutaneous emphysema, pneumothorax, and pneumomediastinum. ${ }^{3}$ Isolated tracheal injury or main bronchial rupture limited to the mediastinum results in massive mediastinal and deep cervical emphysema, without pneumothorax (type 2 injury). Extramediastinal bronchial rupture with communication to the pleural space causes ipsilateral pneumothorax and most often associated pneumomediastinum (type 1 injury). ${ }^{2}$

Immediate primary repair of the bronchial rupture is advocated to preserve functional lung tissue and to provide the best long-term results. ${ }^{1,2,4}$ Resection of lung tissue, however, cannot always be avoided.

Symbas, Justicz, and Ricketts ${ }^{5}$ have divided tracheobronchial ruptures into three types: transverse, longitudinal, and complex, complex injuries being either combined transverse and longitudinal ruptures or multiple ruptures. In their review of 189 cases, complex ruptures represented only $8 \%$ of the total. In the two patients with involvement of a lobar bronchus, a lobe resection was performed. In our patient, full functional preservation of the right lower lobe was obtained by primary repair of the ruptured lobar bronchus.

Most intrathoracic airway ruptures caused by blunt 

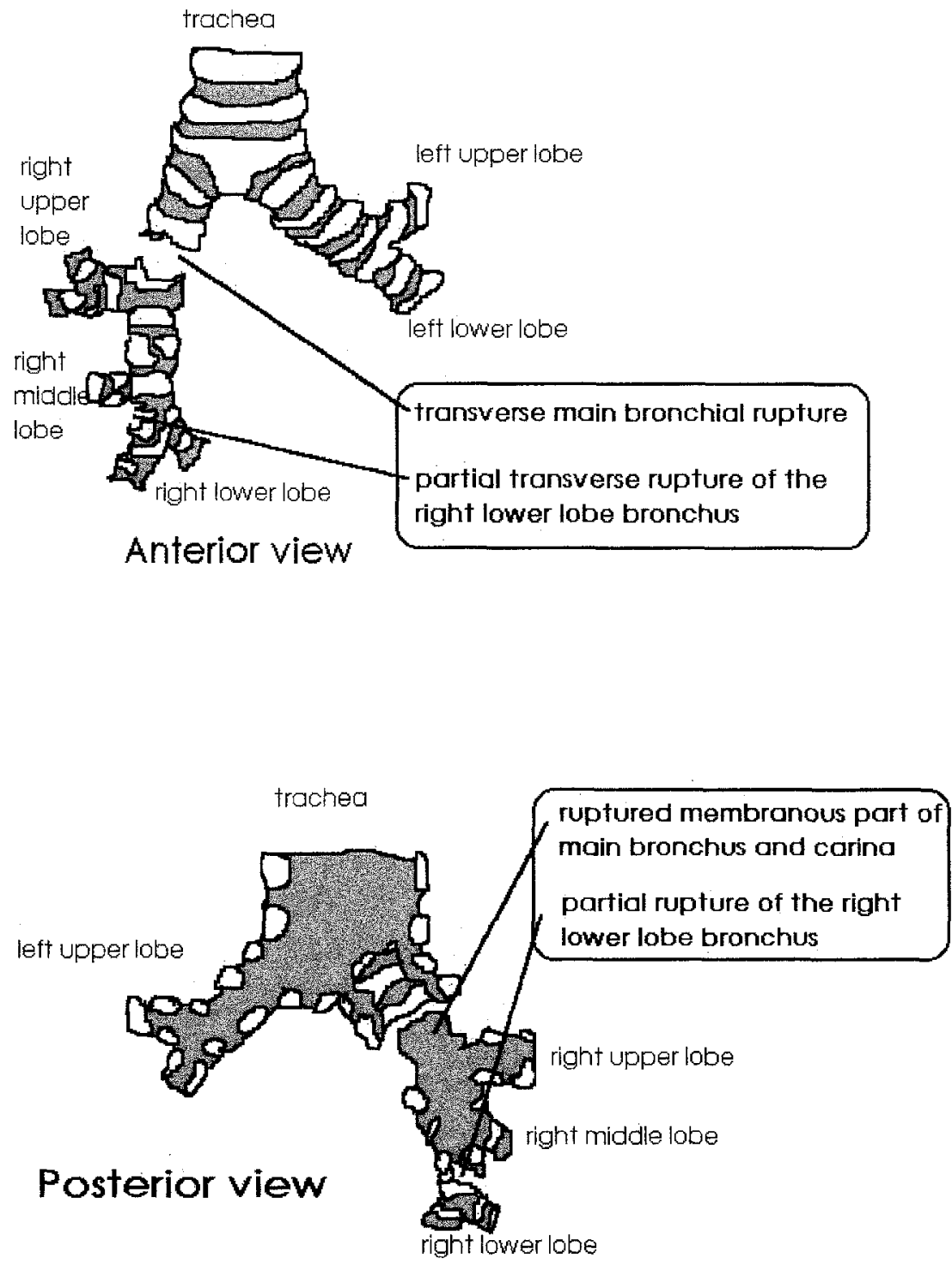

Fig. 1. Anterior and posterior views of a rupture of the right main bronchus and the right lower lobe bronchus.

trauma are situated at the distal trachea or the main bronchi within $2.5 \mathrm{~cm}$ of the carina. ${ }^{2,4}$ This was true of the right main bronchus and carinal injury in our patient.

Cardiopulmonary bypass has been used for the repair of complex bilateral ruptures. ${ }^{5}$ An important disadvantage is the risk of generalized heparinization, with possible bleeding at other sites of injury such as fractures or cerebral lesions.

Anesthetic management of the ruptured airway can be difficult and hazardous. Care should be taken to prevent further disruption of the airway during endotracheal intubation. Bronchoscopic guidance must be used. The tube should be passed distal to the rupture in the case of tracheal rupture, and it should be passed into the main bronchus of the unaffected lung in the case of bronchial rupture. $^{2,5}$

The prognosis of tracheobronchial ruptures is often related to the severity of associated injuries in these multiply injured patients. Over the long term, bronchial stenosis by granulation tissue after primary repair of a bronchial rupture is to be feared. ${ }^{3}$ Regular bronchoscopic checks should be performed. Stenosis can be managed either with endoscopic laser therapy or, if endoscopic treatment is unsuccessful, by reoperation.

In conclusion, in this patient with a complex tracheobronchial rupture, primary repair of the bronchus was possible even at the level of a lobar bronchus, with complete functional preservation of lung tissue. 


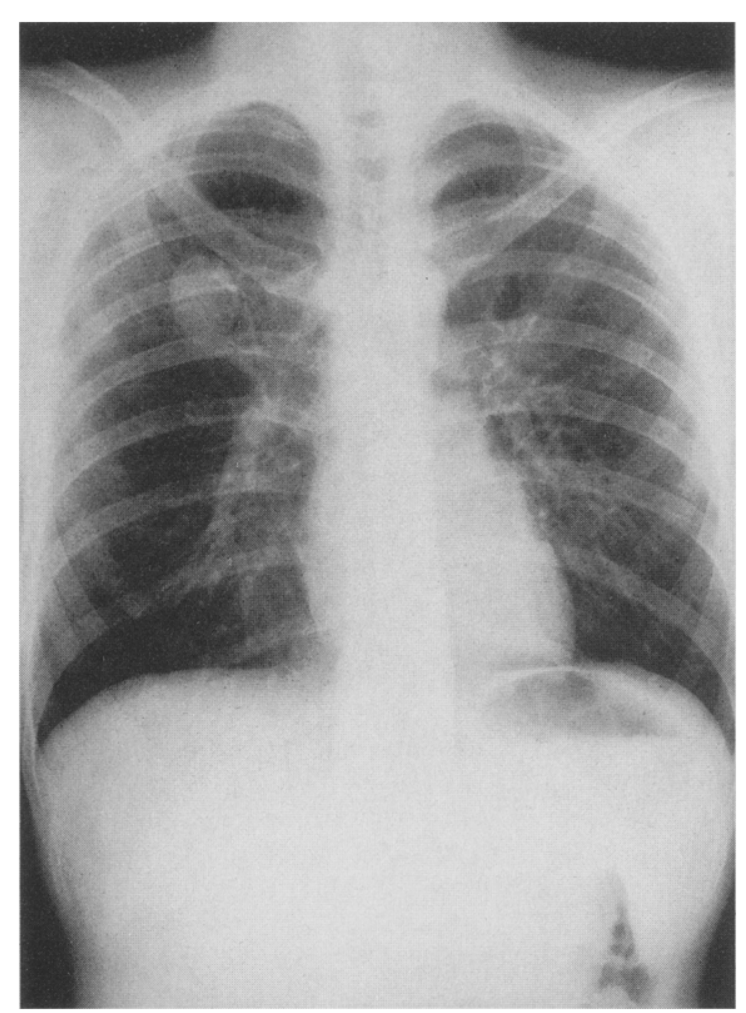

Fig. 2. Roentgenogram of the chest 30 days after repair. A residual hematoma of the right upper lobe and good expansion of the right lower lobe can be seen.

\section{REFERENCES}

1. Baumgartner F, Sheppard B, de Virgilio C, et al. Tracheal and main bronchial disruptions after blunt chest trauma: presentation and management. Ann Thorac Surg 1990:50: 569-74.

2. Mulder DS, Ratnani S. Tracheobronchial trauma. In: Pearson FG, Deslauriers J, Ginsberg RJ, Hiebert CA, McKneally MF, Urschel HC, editors. Thoracic surgery. 1st ed. New York: Churchill Livingstone, 1995:1543-54.

3. Spencer JA, Rogers CE, Westaby S. Clinico-radiological correlates in rupture of the major airways. Clin Radiol 1991:43: 371-6.

4. Hartley C, Morrit GN. Bronchial rupture secondary to blunt chest trauma. Thorax 1993:48:183-4.

5. Symbas PN, Justicz AG, Ricketts RR. Rupture of the airways from blunt trauma: treatment of complex injuries. Ann Thorac Surg 1992:54:177-83.

\section{HLA ANTIBODIES SPECIFIC FOR CRYOPRESERVED HEART VALVE "HOMOGRAFTS" IN CHILDREN}

Inez den Hamer, MD, ${ }^{a}$ Bouke Hepkema, PhD, ${ }^{b}$ Jochum Prop, MD, ${ }^{\text {a }}$ Nynke Elzenga, MD, ${ }^{\mathrm{c}}$ and Tjark Ebels, MD, Groningen, The Netherlands

For more than 30 years, human heart valves have been used as grafts for replacement of diseased aortic and pulmonary valves. These heart valve allografts, tradition-

From the Departments of Cardiopulmonary Surgery, ${ }^{a}$ Transplantation Immunology, ${ }^{b}$ and Pediatric Cardiology, ${ }^{c}$ University Hospital Groningen, Groningen, The Netherlands.

Received for publication March 22, 1996; accepted for publication May 3, 1996.

Address for reprints: Jochum Prop, MD, Cardiopulmonary Surgery, Research Division, University Hospital Groningen, P.O. Box 30.001, 9700 RB Groningen, The Netherlands.

J Thorac Cardiovase Surg 1997;113:417-9

Copyright (C) 1997 by Mosby-Year Book, Inc.

$0022-5223 / 97 \$ 5.00+0 \quad \mathbf{1 2 / 5 4 / 7 4 7 0 8}$ ally referred to as "homografts," were long assumed to be immunologically inert and were supposed not to be affected by rejection, in contrast to organ transplants. However, in children, degeneration of the heart valve allografts is frequently observed. ${ }^{1}$ Findings in clinical ${ }^{2,3}$ and experimenta ${ }^{4}$ studies suggested that this degeneration might be caused by an immune response. Recently, Smith and colleagues ${ }^{5}$ from the Harefield Hospital, United Kingdom, found donor HLA-specific antibodies in adults after implantation of fresh or antibiotic-sterilized aortic valve allografts. This demonstrated that human heart valve allografts can induce immune responses in patients.

Most cardiac centers do not have fresh heart valve allografts available and use cryopreserved valves instead, which have been shown to become less immunogenic during the cryopreservation procedure. ${ }^{3}$ Therefore we investigated whether cryopreserved heart valve allografts induced donor-specific HLA antibodies in children. 